\title{
High-order perturbative expansions in massless gauge theories with NSPT
}

\author{
Luigi Del Debbio ${ }^{1}$, Francesco Di Renzo², and Gianluca Filaci ${ }^{1, \star}$ \\ ${ }^{1}$ Higgs Centre for Theoretical Physics, School of Physics and Astronomy, University of Edinburgh, Edinburgh \\ EH9 3FD, United Kingdom \\ ${ }^{2}$ Dipartimento di Fisica e Scienze della Terra, Università di Parma and INFN, Gruppo Collegato di Parma \\ I-43100 Parma, Italy
}

\begin{abstract}
We investigate the possibility of using numerical stochastic perturbation theory (NSPT) to probe high orders in the perturbative expansion of lattice gauge theories with massless Wilson fermions. Twisted boundary conditions are used to regularise the gauge zero-mode; the extension of these boundary conditions to include fermions in the fundamental representation requires to introduce a smell degree of freedom. Moreover, the mass of Wilson fermions is affected by an additive renormalisation: we study how to determine the mass counterterms consistently in finite volume. The knowledge of the critical masses will enable high-order perturbative computations in massless QCD, e.g. (as a first application) for the plaquette.
\end{abstract}

\section{Introduction}

Numerical stochastic perturbation theory (NSPT) is a technique which allows to perform perturbative expansions numerically in a quantum (field) theory. Here, in particular, we are interested in applying NSPT to a $S U\left(N_{c}\right)$ lattice gauge theory in four dimensions with massless Wilson fermions in the fundamental representation, see [1] for a review. When sufficient high orders are reached, it is possible to study the divergent behaviour of a perturbative series: the pattern of divergence (e.g. renormalons) gives information on non-perturbative physics (e.g. power corrections in the OPE). There are wellestablished results in lattice gauge theories only for gluodynamics [2-4]. In fact fermions are a handle on the beta function, they affect the running of the coupling and should control and determine the high-order perturbative behaviour.

\section{Twisted boundary conditions and smell}

When a theory is defined in finite volume, the fields can have boundary conditions compatible with all the symmetries of the action. We want to adopt twisted boundary conditions in order to remove the gauge zero-mode (that would spoil NSPT convergence) and have a well-defined perturbative expansion in finite volume. Denoting $U_{\mu}(x) \in S U\left(N_{c}\right)$ a link starting at site $x$ in direction $\hat{\mu}$, twisted

\footnotetext{
^e-mail: g.filaci@ed.ac.uk
} 
boundary conditions [5] in direction $\hat{v}$ are

$$
U_{\mu}(x+L \hat{v})=\Omega_{v} U_{\mu}(x) \Omega_{v}^{\dagger},
$$

where $\Omega_{\mu} \in S U\left(N_{c}\right)$ are a set of matrices satisfying

$$
\Omega_{v} \Omega_{\mu}=z_{\mu \nu} \Omega_{\mu} \Omega_{v}, \quad z_{\mu \nu} \in Z_{N_{c}} .
$$

Including fermions in the fundamental representations is not straightforward: the same gauge transformation for the fermions reads

$$
\psi(x+L \hat{v})=\Omega_{v} \psi(x)
$$

and implies an ambiguous evaluation of $\psi(x+L \hat{\mu}+L \hat{v})$. An idea, proposed in [6], to overcome this problem is to introduce a new quantum number so that fermions exist in different copies, or smells, which transform into each other according to the antifundamental representation of $S U\left(N_{c}\right)$. The theory has a new global symmetry, but physical observables are singlets under the smell group. Thus, configurations related by a smell transformations are equivalent and we are free to substitute eq. (3) with

$$
(\psi(x+L \hat{v}))_{c, s}=\sum_{c^{\prime}, s^{\prime}}\left(\Omega_{v}\right)_{c c^{\prime}}(\psi(x))_{c^{\prime}, s^{\prime}}\left(\Lambda_{v}^{\dagger}\right)_{s^{\prime} s}
$$

where $c, s$ stand respectively for colour and smell indices and $\Lambda_{v} \in S U\left(N_{c}\right)$. It is useful to think of the fermion field as a matrix in smell-colour space. If the transformation matrices in smell space satisfy the same relations as in eq. (2) (in particular we choose them to be equal to the $\Omega$ s), then twisted boundary conditions are well-defined.

In the stochastic process, the drift due to the fermions has to be multiplied by $N_{f} / N_{c}$ in order to have $N_{f}$ flavours in the infinite-volume limit. In other words, this implies that we are taking the $N_{c}$ th root of the Wilson fermion determinant and that we are dealing with a nonlocal action, because twisted boundary conditions break the invariance under smell transformations. Despite that, it is possible to show, with arguments similar to those presented in [7], that perturbatively this operation is legitimate and leads to a renormalisable theory.

Boundary conditions determine momentum quantisation. For the gauge field, we investigated twisted boundary conditions on a plane (i.e. directions $\hat{1}, \hat{2}$ are twisted, while $\hat{0}, \hat{4}$ are periodic) and triple twist (i.e. directions $\hat{1}, \hat{2}, \hat{3}$ are twisted, while $\hat{0}$ is periodic). The fermion field transforms accordingly, but direction $\hat{0}$ is always antiperiodic. With this setup, colour and momentum degrees of freedom mix in Fourier space: the transform of a matrix field ${ }^{1}$ in position space is a scalar function of the momenta. Of course, not to lose degrees of freedom, momentum has a finer resolution compared to the one with periodic boundary conditions: now it is

$$
\frac{2 \pi}{L}\left(n_{0}+\frac{\eta}{2}, n_{1}+\frac{\tilde{n}_{1}}{N_{c}}, n_{2}+\frac{\tilde{n}_{2}}{N_{c}}, n_{3}+\frac{\tilde{n}_{3}}{N_{c}}\right),
$$

where

$$
\begin{aligned}
n_{0}, n_{1}, n_{2}, n_{3} & =0, \ldots, L-1 \\
\tilde{n}_{1}, \tilde{n}_{2}= & 0, \ldots, N_{c}-1 \\
\tilde{n}_{3} & =\left\{\begin{array}{l}
0 \text { if the twist is on a plane } \\
\text { a value between } 0 \text { and } N_{c}-1, \text { fixed by } \tilde{n}_{1} \text { and } \tilde{n}_{2}, \text { for triple twist }
\end{array}\right. \\
\eta & =\left\{\begin{array}{l}
0 \text { if direction } \hat{0} \text { is periodic (gluon momentum) } \\
1 \text { if direction } \hat{0} \text { is antiperiodic (fermion momentum) }
\end{array}\right.
\end{aligned}
$$

\footnotetext{
${ }^{1}$ We deal with matrices in colour space, like the gauge potential, or matrices in colour-smell space, like the fermion field.
} 
The traceless property of the gauge potential corresponds to the absence of degrees of freedom with $\tilde{n}_{1}=\tilde{n}_{2}=0$. The fast Fourier transform in our code has been efficiently extended to perform Fourier transforms in smell-colour space.

In order to have full control on our implementation, we checked lowest order results of the simulations against finite-volume predictions in twisted lattice perturbation theory, introduced in [8]. Feynman rules are fairly similar to lattice perturbation theory, apart from some overall factors and phases in propagators and vertices; all phases happen to cancel in the first-order computations we considered. The major difference is the sum over momenta inherited by the quantisation,

$$
\sum_{k}=\sum_{n_{1}=0}^{L-1} \sum_{n_{2}=0}^{L-1} \sum_{n_{3}=0}^{L-1} \sum_{n_{4}=0}^{L-1} \sum_{\tilde{n}_{1}=0}^{N_{c}-1} \sum_{\tilde{n}_{2}=0}^{N_{c}-1}
$$

where $k$ is quantised according to eq. (5). When the sum involves a gluon momentum, all terms with $\tilde{n}_{1}=\tilde{n}_{2}=0$ are removed. Each fermion loop has to be divided by $N_{c}$, i.e. by the numbers of smells running in the loop.

An interesting quantity to be studied is the $1 \times 1$ Wilson loop (plaquette)

$$
W=\frac{1}{N_{c}}\left\langle\operatorname{Tr} U_{P}\right\rangle=1+\frac{1}{\beta} W^{(1)}+\frac{1}{\beta^{2}} W^{(2)}+\ldots,
$$

where $U_{P}$ is the product of four links and $\beta=2 N_{c} / g^{2}, g$ is the Yang-Mills coupling. The contribution from the plaquettes lying on the directions $\hat{\mu}$ and $\hat{v}$ is

$$
W_{\mu \nu}^{(1)}=-\frac{1}{2 L^{4}} \sum_{k} \frac{\hat{k}_{\mu}+\hat{k}_{\nu}}{\hat{k}^{2}},
$$

so that $W^{(1)}=\frac{1}{6} \sum_{\mu>v} W_{\mu \nu}^{(1)}$. This expression coincides with the one presented in [9]. The agreement between the analytic predictions and our measures can be seen in Figure 1.

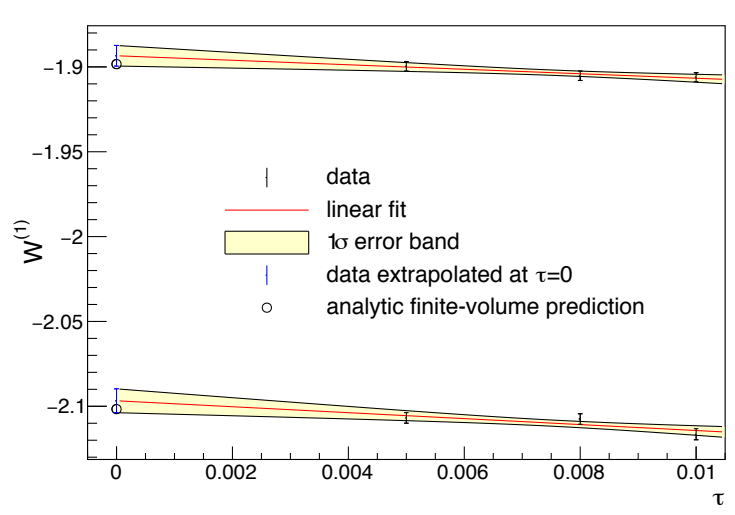

Figure 1. Measure of $W^{(1)}$ for a $2^{4}$ lattice with triple twisted boundary conditions at three different time-steps $\tau$ of the stochastic process and first-order integrator, $N_{c}=3$. The lower measures correspond to the average of the plaquette in the three planes identified by two twisted directions, the upper ones correspond to the average in the three planes identified by one twisted direction and one periodic direction; the size of the lattice has been chosen to enhance the difference of the plaquette value between these two groups of planes.

\section{Critical mass}

The inverse of the Wilson fermion propagator in momentum space can be expressed as

$$
a S(a p)^{-1}=i \sum_{\mu} \gamma_{\mu} \overline{\left(a p_{\mu}\right)}+\frac{1}{2} \widehat{(a p)}^{2}+a m+a \Sigma\left(a p, a m, \beta^{-1}\right),
$$


where $\bar{v}_{\mu}=\sin v_{\mu}, \hat{v}_{\mu}=2 \sin \left(\frac{v_{\mu}}{2}\right), m$ is the bare mass of the fermion and $a$ is the lattice spacing. The self energy $\Sigma\left(a p, a m, \beta^{-1}\right)$ is the one-particle irreducible fermion two-point function and is proportional to the inverse lattice spacing $a^{-1}$. This function has to be renormalised in order to kill the divergence that would arise in the continuum limit. We adopt the on-shell renormalisation scheme: in the massless case, it follows that

$$
a m+\Sigma\left(a p=0, a m, \beta^{-1}\right)=0 .
$$

As observed in [10], this prescription matches the one obtained by requiring the chiral Ward identity to hold in the continuum limit.

Wilson fermions are not equipped with chiral invariance when $m=0$ and eq. (10) does not hold for free: the self energy at zero momentum is affected by a power divergence $a^{-1}$, which has to be cured by an additive renormalisation. The critical mass $m_{c}$ is defined to be the value of the bare mass such that the renormalised mass of the fermion is zero. Expanding eq. (10) in the coupling defines the critical mass $m_{c}=\sum_{k} m_{c}^{(k)} \beta^{-k}$ order by order in perturbation theory.

With the goal of simulating a massless theory, the knowledge of these mass counterterms becomes extremely important. In NSPT it is possible to generate gauge field configurations distributed according to $\exp \left(-S_{g}[U]+N_{f} \operatorname{Tr} \log M[U]\right)$, where $S_{g}[U]$ is the Wilson gauge action and $M[U]$ is the Wilson Dirac operator; the gauge field is treated as an expansion in the coupling, $U=1+\sum_{k} U^{(k)} \beta^{-k / 2}$. This allows to measure the perturbative expansion of the fermion propagator and infer the critical mass,

$$
S(p)=\langle\psi(p) \bar{\psi}(-p)\rangle=\int \mathcal{D} U e^{-S_{\mathrm{g}}[U]+N_{f} \operatorname{Tr} \log M[U]} M[U]_{p p}^{-1} .
$$

Our strategy is to collect $N$ configurations $\left\{{ }^{(n)} U_{\tau}, n=1, \ldots, N\right\}$ for different time steps $\tau$ of the stochastic process; for each one the Landau gauge is fixed as in [11]. Then the inverse Dirac operator is applied to a delta function in momentum space,

$$
S(p)_{\alpha \beta, \tau}^{(n)}=\sum_{p \gamma} M\left[{ }^{(n)} U_{\tau}\right]_{p q, \alpha \gamma}^{-1} \delta_{p q} \delta_{\gamma \beta},
$$

where $\alpha, \beta$ are Dirac indices; autocorrelation effects are evaluated according to [12]. It is important to stress again that, because of twisted boundary conditions, the fermion propagator in momentum space is not a colour matrix anymore. The average of ${ }^{(n)} S(p)_{\tau}$ over all the configurations gives our Monte Carlo estimate of $S(p)_{\tau}$. We can now extrapolate the stochastic time step to zero, $S(p)_{\tau} \stackrel{\tau \rightarrow 0}{\longrightarrow} S(p)$ and invert the propagator to obtain $S(p)^{-1}$. Finally, the inverse propagator is projected onto the identity in Dirac space, $\Gamma(a p)=\frac{1}{4} \operatorname{Re} \operatorname{Tr} a S(a p)^{-1}$, and the critical mass is given by the value of $\Gamma(a p)$ at $p=0$. All these operations are performed order by order in perturbation theory keeping in mind that, after the measure of the propagator, all perturbative orders $\beta^{-k / 2}$ with an odd $k$ are discarded, since the expansion in powers of $\beta^{-1 / 2}$ is an artefact of NSPT.

With this procedure, it is possible to measure the critical mass at a certain order $\bar{k}$ if the critical masses at orders less than $\bar{k}$ are known and the set of configurations is generated keeping those into account. $\Gamma(a p)^{(k)}$ will extrapolate to zero for all orders $k<\bar{k}$ and the extrapolated value at order $\bar{k}$ will give $m_{c}^{(\bar{k})}$. In order to move forward and compute the next order, a new set of configurations where $m_{c}^{(\bar{k})}$ is taken into account is needed.

\section{Zero-momentum extrapolation and valence twist}

On the lattice, we are allowed to measure $\Gamma(a p)$ only for the momenta given by eq. (5); consequently, a good functional form is needed to extrapolate our data to zero momentum. 

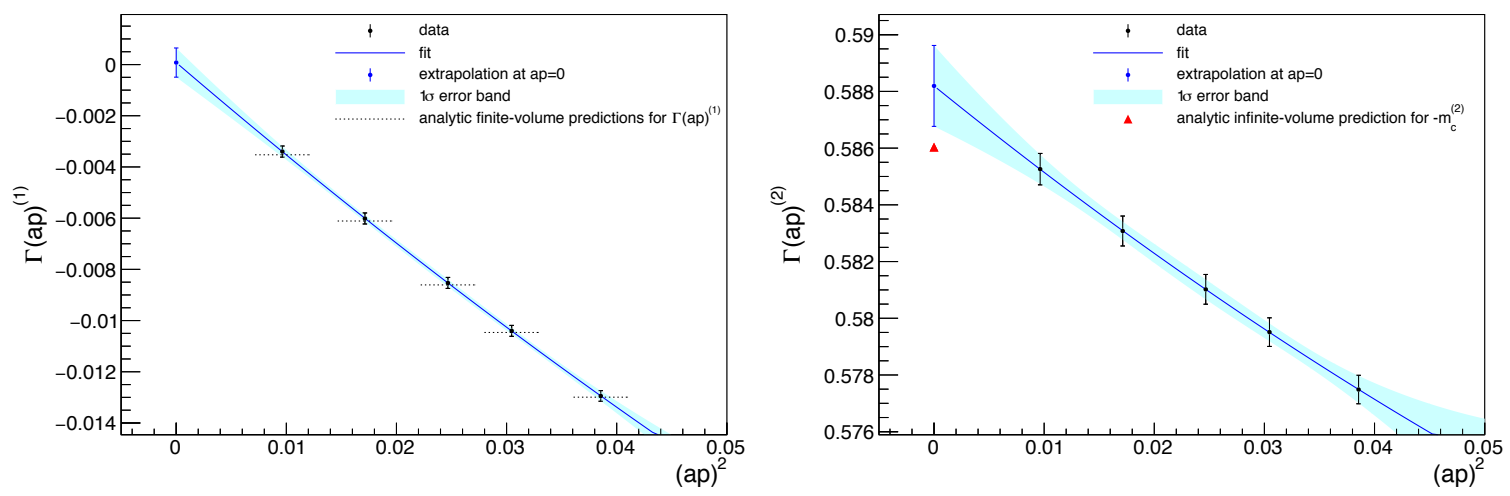

Figure 2: Measure of $\Gamma(a p)^{(1)}$ (left panel) and $\Gamma(a p)^{(2)}$ (right panel) for a $12^{4}$ lattice with twisted boundary conditions on a plane, $N_{c}=2, N_{f}=2$. The analytic finite-volume critical mass $m_{c}^{(1)}$ is included in the simulation. A second-order polynomial in $(a p)^{2}$ is used for fitting. Most analytic finite-volume predictions have been drawn as lines to help the eye in the comparison.

The strategy adopted in literature (for example in [13]) has been an expansion in the lattice spacing: in the infinite-volume limit, it leads to a hypercubic symmetric Taylor expansion composed of invariants in $a p$, logarithms of $a p$ and ratios of invariants; an explicit computation to order $a^{2}$ is shown in [14]. The ratios and the logarithms arise because of the infrared divergences that appear deriving the integrands respect to $a$. On the other hand, working in a consistent way in finite volume does not cause any infrared divergence: expressions for $\Gamma(a p)^{(k)}$ will be just sums of ratios of trigonometric functions, which we can expand in $a$ obtaining simply a combination of polynomial lattice invariants ${ }^{2}$. Still, this is not enough to perform a reliable extrapolation: from the analytic expansion of $\Gamma(\text { ap })^{(1)}$, we realised that even the lowest momentum allowed, $\frac{\pi}{L}$, is far from the convergence region of this series. This happens for reasonably big lattices, $L \lesssim 32$.

Now the key observation is that the boundary conditions of the "valence fermions" (i.e. the boundary condition of the inverse Dirac operator in eq. (11)) are arbitrary: in principle, setting their boundary conditions to be periodic could give us directly the critical mass. Unfortunately that is not possible, because we do not have direct access to $\Gamma(a p)$ but only to the propagator, which is tree-level divergent at $p=0$. Despite that, switching from antiperiodic to $\theta$-boundary conditions in the $\hat{0}$ direction for the valence fermions,

$$
\psi(x+L \hat{0})=e^{i \theta} \psi(x),
$$

allows to span a number of momenta $p_{0}=\frac{\theta}{L}$ which are within the convergence radius of the $a$ expansion. The hypercubic series becomes a polynomial in $p_{0}^{2}$. We can use that to fit the data and extract a solid estimate of the critical mass in finite volume, as in Figure 2.

We also computed analytically the one-loop trace of the inverse of the fermion propagator in twisted lattice perturbation theory: the agreement with our simulations is extremely good, see again Figure 2. We also verified at this order that our renormalisation prescription is gauge-invariant (result true at all orders thanks to the gauge-invariance of the pole mass, proven in [15]).

\footnotetext{
${ }^{2}$ Expanding in $a$ and sending the lattice size to infinity are operations that do not commute; this gives rise to different series in the finite- and infinite-volume cases.
} 

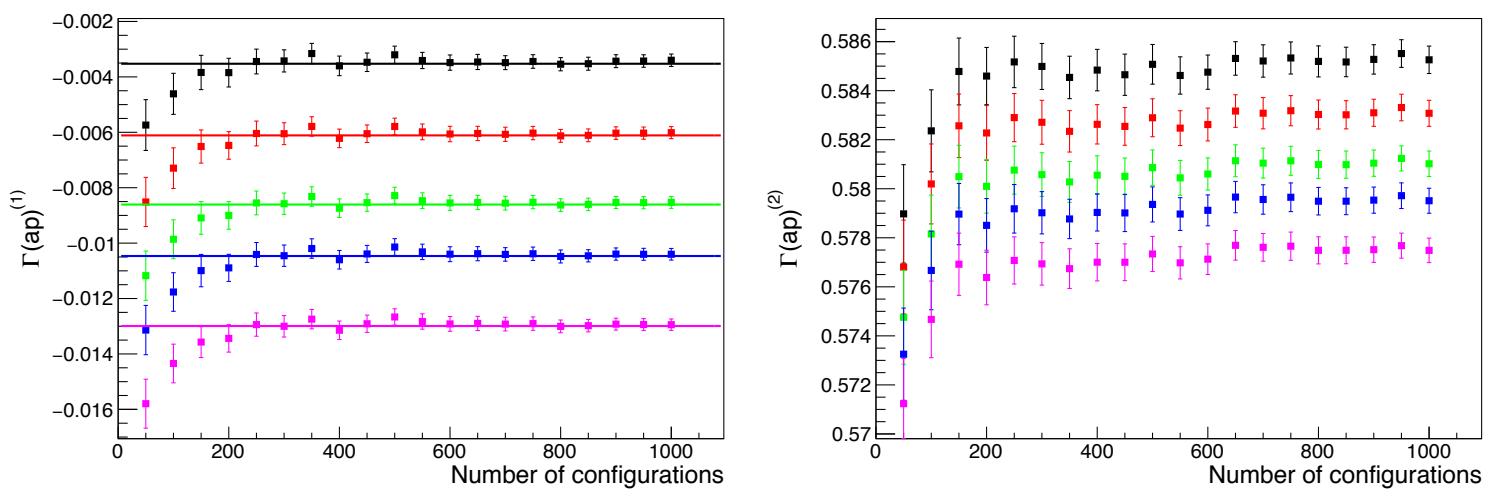

Figure 3: Dependence of the data shown in Figure 2 on the number of configurations included in the analysis. Left panel corresponds to $\Gamma(a p)^{(1)}$ data, right panel to $\Gamma(a p)^{(2)}$. Different colours correspond to different momenta. Horizontal lines are the analytic finite-volume predictions.

Moreover, it has to be stressed that measuring such low momenta requires a careful analysis of the thermalisation: this is evident from the plots in Figure 3. At the lowest order we can check directly when the measures agree with the theoretical predictions; at higher orders, it is important to wait until the measures stay stable. A few hundreds uncorrelated configurations are required.

\section{Conclusion}

We have implemented NSPT for SU(2) and SU(3) gauge theories with an arbitrary number of fermions (with smell) in the fundamental representation and with twisted boundary conditions. We also developed a way to extract reliably the mass counterterms in finite volume and therefore keep the Wilson fermion mass under control. All the measures have been found in very good agreement with one-loop twisted lattice perturbation theory predictions. With this setup, it will be possible to start and collect the critical masses and study, for example, high-orders perturbative expansions in full QCD.

\section{References}

[1] F. Di Renzo, L. Scorzato, JHEP 10, 073 (2004), hep-lat/0410010

[2] C. Bauer, G.S. Bali, A. Pineda, Phys. Rev. Lett. 108, 242002 (2012), 1111.3946

[3] G.S. Bali, C. Bauer, A. Pineda, C. Torrero, Phys. Rev. D87, 094517 (2013), 1303.3279

[4] G.S. Bali, C. Bauer, A. Pineda, Phys. Rev. D89, 054505 (2014), 1401.7999

[5] G. 't Hooft, Nucl. Phys. B153, 141 (1979)

[6] G. Parisi, Prolegomena to any future computer evaluation of the QCD mass spectrum, in Proceedings of a NATO Advanced Study Institute on Progress in Gauge Field Theory: Cargèse, Corsica, France (1984), pp. 531-541

[7] S.R. Sharpe, PoS LAT2006, 022 (2006), hep-lat/0610094

[8] A. González-Arroyo, M. Okawa, Phys. Rev. D27, 2397 (1983)

[9] M.G. Pérez, A. González-Arroyo, M. Okawa (2017), 1708.00841

[10] M. Bochicchio, L. Maiani, G. Martinelli, G.C. Rossi, M. Testa, Nucl. Phys. B262, 331 (1985) 
[11] P. Rossi, C.T.H. Davies, G.P. Lepage, Nucl. Phys. B297, 287 (1988)

[12] M. Lüscher, Comput. Phys. Commun. 165, 199 (2005), hep-lat/0409106

[13] F. Di Renzo, V. Miccio, L. Scorzato, C. Torrero, Eur. Phys. J. C51, 645 (2007), hep-lat/0611013

[14] M. Constantinou, V. Lubicz, H. Panagopoulos, F. Stylianou, JHEP 10, 064 (2009), 0907.0381

[15] A.S. Kronfeld, Phys. Rev. D58, 051501 (1998), hep-ph/9805215 